\title{
Detect-caused Fire and Bang Alarm using Image Processing and Sensors
}

\author{
Kamini Sharma ${ }^{1}$, Dolly Jadhwani ${ }^{2}$ \\ Department of Computer Engineering, Government College of Engineering, Jalgaon ${ }^{1,2}$
}

\begin{abstract}
Detection of flame and its classified category is the most demanding and testing work in processing of the image. The use of image processing without the use of sensors helps to detect the fire in more precise, more accurate and in the fast manner. Hence the proposed paper is trying to introduce the procedure towards the optimum solution for the detection of flame as early as possible. In order to make the action less in amount in the loss of human and loss in property as well, an intelligent fire detection system is been proposed so as to detect flame with higher accuracy.
\end{abstract}

Keywords: fire detection; color segmentation; image processing.

\section{INTRODUCTION}

In many flame detection systems, the systems are based on the sensors so as to make a little nearer to completely accurate decision. The recent trend of available sensors which are used: such as smoke detector, warmth detector, fire detector, these all take lot of time to response to be detected, also these detectors mentioned above cannot identify, analyze or visualize the damage so caused. These mentioned sensors need to be placed in various positions or places carefully because they are not appropriate for the places that are exposed. This fire detection is done by using software that processes the outputs of CCTV cameras in real time. Current vision systems are based on color clues, motion in fire and edge detection of flame. There is rapid and fast development in the technology of flame detection and in addition to them video processing are the new flame detection conventional technique which is soon going to be substituted by the computer vision based system so as to make the work easier and eliminate the loss. The flame detectors are widely used in buildings along with the conventional point smoke detectors. Smoke and fire detectors are the one which typically recognize the certain particles that are present and generated by smoke and fire by using the concept of ionization or photometry. Alarm is not issued unless the particles reach the sensor to activate them. Hence forth, their use is not possible in open space and large areas. In addition to the smoke detectors, closed circuit television (CCTV) surveillance systems are at present made in ready to use in large number of public places in monitoring the indoors and outdoors action of putting things to transform into the operation. Such systems may gain an early fire detection capability.The National Fire Protection Association (NFPA) is a standard that has been described exactly by the nature that by many of different classified classes of flame are present, out of these presen frames the proposed system focuses on finding the different classes like that of class A and class C objects-classified by NFPA. Whereas when we consider class A objects it actually excludes rest of the remaining classes and includes things which include example such as rubber, a sheet of paper, things made up of wooden, clothes or plastic and finally the class $\mathrm{C}$ objects includes electrical objects such as outlet, boards of power, electric motors, appliances etc. The input data given to the system is obviously the real time- dynamic local shooting video i.e. generated from any video considerably the cameraFire scheme of detection, this can be made more robust if identified the pixels of grey cycle pixels which are in neighboring to the flame and measuring flame area

This finding is probably based on two of the figures of merit; that are (i) shape of the region and (ii) the minimal changes of the region. The flexibility of flame detector finely depends on highly on flame pixel classifying the robustness to which it prepares root areas, to which the remaining system gets executed. The categorization of pixels of flame is therefore very vital to get produced with a very high accuracy of detection and also lower down the percentage if false alarm so produced. The fire pixel scheme categorization is considered in color video sequences and as well as the scale of grey. Usage of the video in this makes the criteria set and get possible to let know the huge and places that are open. The system will be demarcated by the position of flame by not excluding that area which is superimposed in a bright red colour box.

\section{RELATED WORK}

Flame detection is an issue which becomes vital and also is related to the human being life and also is concerned to security. Image processing is actually the based system that completes the very fast detection, just as particularly the human eyes does, i.e. depending on working of camera.

Here there are 4 ways in which the fire can be put out they are as follows:

- The most regularly in use burning material, Coal

- Excluding the oxygen 
-To Remove the fuel

- And Break the chemical reaction [5]

Today in this era of fast technology the flame detection systems are based on detecting smoke, gas and flame and depend on sensors. However sensors are not smart thing to do this job because of its speed. It depends on distance between fire area and sensor location. Earlier systems were based only on sensor. There are many fire detection systems which are based on video imaging that are implemented in different research papers, with excluding some of detection which uses video sequencing. The fire detection research is generally based on video sequences and is divided into two following categories: fire flame detection and fire smoke detection [1]. Proposed system connected to fire flame detection technique, therefore it is discussed in this section.

Han et. $\mathrm{Al}$ [1] the flame detection algorithm was divided into two categorized algorithms and they are detection of fire and detection of smoke. While one of the researcher Han et. al. [1] used features like color and motion on other hand Kandil et al. [3] and M. Nixon, A. Aguando [6] made use of shape and color features to analyze an appearance of fire.Tareyinet.al [2] was the one who used the features that were extracted such as movment, flickering of fire, edge blurring region of fire from a video, this all was done using the wavelet transformation and subtraction of the backgroud so as to determine the smoke produced from the fire caused. Kandilet.al [3] and Liu et.al[4] were the two researchers that used different figure shapes and color features to analyze occurrence of flame. Among many of these researchers, they used different properties of flame which were no unusual such as color, movement, edges, shapes of the fire. Lai et al. [5] advised, features of flame caused can be make in use for detection in early stages of fire. Lai introduced development of automatic video surveillance systems has been the core trend in the security and guard service industry. This proposed paper demonstrates real time video contents analysis, a very simple criteria for detecting the fire and its destruction event automatically in the area so kept under the observation. Not slow but fast and approximately exact detection execution which is so developed for early causing of flame alert purpose to the surrounding therefore to subtract the loss caused by fire accidents by observing features of fire event. This proposed paper view that the proposed algorithm is not only achieves real-time requirement and has better performance than the compared surveillance systems, but also attains the goal of alleviating the existing system cost efficiently [5]. Zhu cascade rejection approach with the Histograms of Oriented Gradients (HoG) features of an image to achieve a fast and accurate human detection system. In HoGs system used variable-size blocks that capture salient features of humans automatically. Ada Boost can be used as feature selection for identifying the appropriate set of blocks from a large set of possible blocks. In this system, integral image of the fire system representation and a ignoring the cascade which significantly increase the speed of the computed outcome. For a configuration of size of $320 \times 280 \mathrm{MP}$ image, the system can compute for the approximately 5 to 30 frames/second. On the dependency of density of scanning picture, while this was approached for maintaining an accuracy method of levels on processing of video so considered as an example. The very first and basic idea proposed in the paper of flame detection is to take a RGB (red, green, blue) model based on chromatic and disorder measurement for taking the fire-pixels out and also get the smoke-pixels extracted. The function for decision of fire-pixels is usually used for reduction by the intensity and saturation of $\mathrm{R}$ component of a picture. The fire- pixels which are extracted from the process will be established,it is the original flame by the growth of dynamic disorder. The ratio which is growing rapidly of flames with respect to a fire caused alarm is purely based on the iteration of checking. This consecutive technique is henceforth a very mind blowing for the examples including the importance at places like military, social security, commercial applications [8]. C.B. Liu introduced his research as the vision (computer) based fire detection. In this proposed system the ratio of increase in the number of CCTV cameras. Computer based fire detection ways produces the advantages over the traditional ways that were complemented the devices so exist. The paper views the spectral, spatial and temporal models of regions of fire in computer based/visual image sequencing. The spectral model was earlier used, representing in terms of the color probability density of fire pixels. The figure made by the fire of a fire region was represented in terms of the spatial frequency content of the region contouring its Fourier coefficients. The consecutive changes at areas in these coefficients are used as the temporal signatures of the fire region. An auto regressive model of the Fourier coefficient series is used. Experiments with a large number of scenes show that the method was capable of detecting fire reliably but there were incorporated in existing surveillance systems at relatively low additional cost [4]

Proposed system uses a simple algorithms based on recognition of color and aloso growth in fire checking. It is a real/run time processing method.

\section{PROPOSED SYSTEM}

\section{A. Methodology}

This system is proposed to uproot a very optimized system so as to analyze an appearance of fire that will be based on images generated by the video. In the paper, mere use of the earlier proposed ways used to conduct the analyzing of the fire and this also proposes a new techniques for the implementation of technique in parallel fashion. By doing so it gives optimized solution for flame to get detected. In the system the following stages are included. The facts that visual color images of fire have high absolute values in the red component of the RGB coordinates are based on algorithm [9]. This property permits simple threshold- 
based criteria on the red component of the color images to Feature:-

segment fire images in natural scenarios. The fire doesn' $t$ 1) Support up to 30 Mega Pixels, the frame rate up to 30 gives high values in the red component. An image is FPS.

loaded into color detection system and mapped with the 2) Excellent quality and fashionable styles.

extracted edge detection image [5]. A color detection 3) Plug and play easy USB interface.

system applies the specific property of RGB pixels and gives the output result as an image with a selected area of color detection.

4) Daily designed to work well both Laptop and Desktop.

\section{B. System Overview}

A well-known device camera is basically used for capturing images of fire. First of all training of the proposed system for recognizing flame, in terms of figures, size, color, and intensity are checked, later the camera clicks the images of that flame or fire, again then it takes the image for the process known color recognition. In this process of color recognition the main focus of concept is that it converts RGB color combination to the GREY and then back from GREY to BINARY code conversion process. Now this will detect or analyse the color combination, mainly it is the due mentioned colors of flame such as red, yellow, orange etc. Making the process to get processed further inputs are taken from sensors which are mentioned above such as smoke sensor and temperature detector. Inputs in form of data from these two different sensors will be helping the human to know more clearly and precisely, and also in identifying the flame. As the identification of fire is done, two different operations are performed. Hence turning ON the pump, here the pump which is considered is the extinguisher and message sending to fire brigade is done depending on the threshold values.

Advantages of the proposed system:-

- Early fire detection and warning.

- RGB color model and image processing provides higher accuracy.

- Assurity of safe environment.

- Installing fire alarms is the early warning benefit

\section{Camera}

A camera or a webcam is a video taking webcam or camera that either streams or feeds its pictures in the real time that is dynamic to or through a personal computer to the proposed computer network i.e. connection.

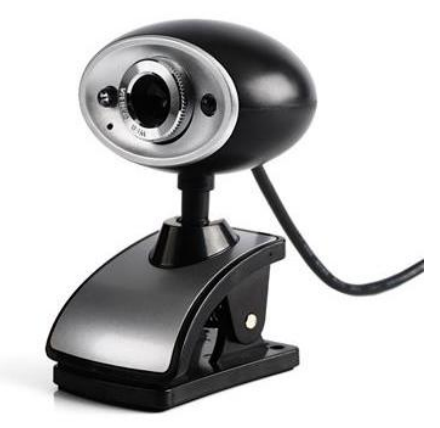

Fig 1:-Camera

\section{Color Recognition}

In fire detection, color is the concept which is playing a very important role in the paper so proposed. Fire has unique and different color values which can be estimated or drawn through the RGB as well as HSI separately. Hardware used are generally displayed or else they are delivered to color via the RGB model. So if a pixel is associated or bounded with a known three dimensional vector i.e.(r, g, b). HSI (Hue, Saturation and Intensity) is an another way of displaying the contents which also follows that how human being see the difference in the flame and its output. Hue, here is representing the preprocessed generated color like orange. Saturation calculates its dilution by white light. HIS does the work of extracting intensity information from the input data, whereas hue and saturation corresponds to human perception. In proposed system, these two color models are considered to retrieve that is to take back the color information of fire flames from different frames.

Following are the different hardware' $s$ which are used for purpose of sensing the fire caused:-

\section{1) Temperature Sensor: LM-35}

The LM35 is an integrated circuit sensor which can be used to measure and analyze the temperature with an electrical output proportional to the temperature (in ${ }^{\circ} \mathrm{C}$ ).

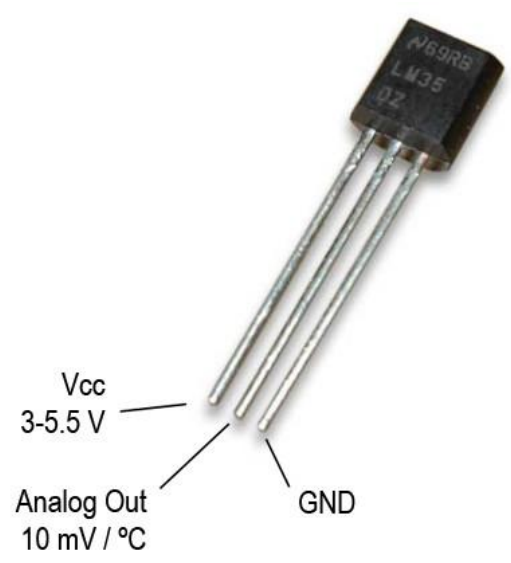

Fig 2:-Temperature sensor (LM35)

\section{2) Smoke Sensor: MQ-2}

The MQ series of gas or the smoke sensors use a small heater inside with a sensor called electro-chemical. They are quite sensitive for a wide range of smoke and are used in indoors which we consider to be at the room temperature. The output so produced is a signal which is basically the analog signal and can be read with an analog input of the Arduino. The MQ-2 Gas Sensor is a module 
which is highly useful for leakage of gas causing smoke
when take the appearance of fire detecting in home and industry

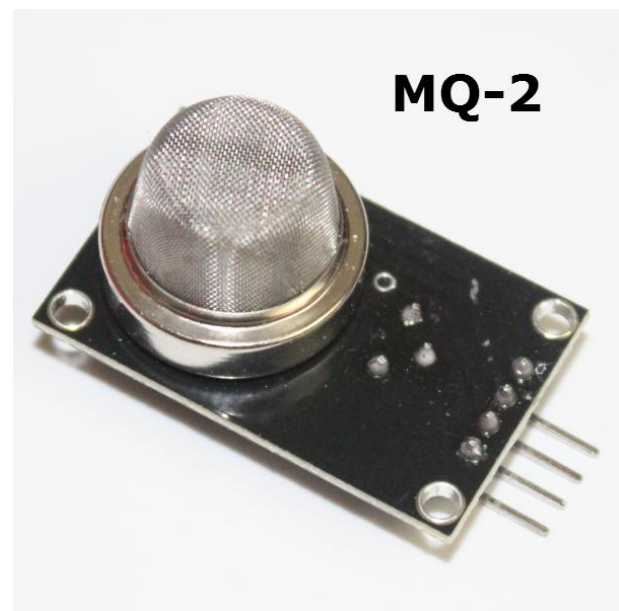

Fig 3:-Smoke sensor (MQ 2)

\section{3) Arduino Board:-}

Arduino is completely an open-source platform background that has been used for making the electronics projects. Arduino also consists of both (i) a physical programmable circuit board (which is often referred to as a microcontroller) (ii) a piece of software, or also an IDE (Integrated Development Environment) that particularly runs on provided computer, it is used to do both the tasks, write and upload the used language code to this Arduino board. The Arduino base has actually came in great demand along with people just as starting out together with the electronics, and for good reasons as well.

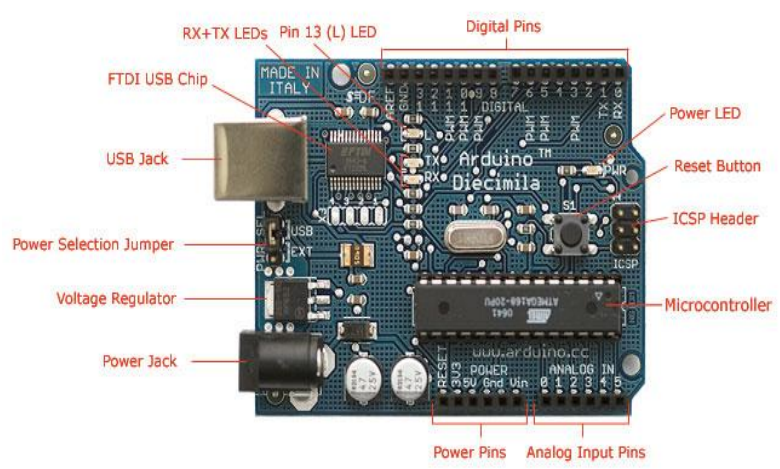

Fig 4:- Arduino Board

\section{ALGORITHMS}

\author{
Algorithm of machine learning \\ 1) Start. \\ 2) Initialize and set the Camera. \\ 3) Capture Frame from surrounding. \\ 4) Turn RGB to Grey. \\ 5) Convert Grey to Binary. \\ 6) Train for fire image. \\ 7) Stop.
}

\section{Algorithms of system}

1) Start.

2) Initialize and set the Camera.

3) Capture Flame.

4) If caused fire is not detected then go to Step 2.

5) Turn RGB to Grey.

6) Transform grey to binary.

7) Detect the Flame.

8) Get readings from smoke sensors (can be temperature sensor).

9) If readings > Threshold then go to 10 .

10) Send a message to fire brigade and turn on the pump.

11) Stop.

\section{Architecture diagram:}

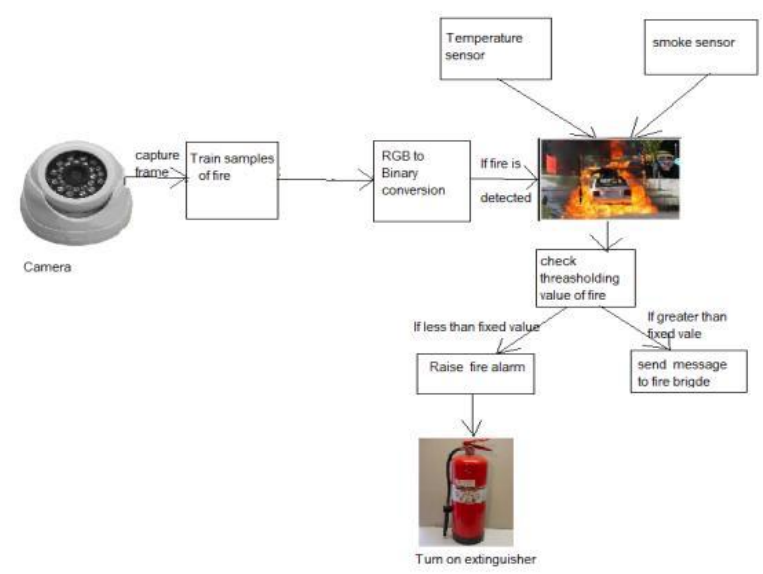

Fig 5:- Architecture diagram of system

\section{CONCLUSION}

The proposed fire detection system has been further improved by considering smoke at early stages of flame. However, detection of smoke is quite challenging task and prone in terms about that, there are high chances of false smoke detections as they can be caused from fog, also the different lighting from cars, street lights etc, climates as well as changes caused in and by nature, and the other outer optical effects.

Such kind of high false flame detections can be solved again by analysing and detecting each and every smokecaused area. However, this tends to a high computational load. In this paper, method adopted of fire detection is based on image processing and sensors. This research is about the developing the automatic fire detection using the RGB color model as well as we are using sensors for accurate result. The stated method is useful for detecting fire automatically and put off it automatically i.e. without manual help the System put off the fire.

\section{FUTURE SCOPE}

In future work, we will also try to analyze the things that would include objects and shapes, such as T-shirts, bags, or other objects with orange color, which are highly 
approached by the camera. The relative proximity caused by change in size and intensity to the camera may cause false alarms. To improve systems fire detection performance Texture or shape information is used along with area information. Along with the fire detection technique the performance of fire pixel can be further improved by applying smoke detection in the early stage of fire. But, detecting smoke is a challenging task and is most likely to cause high false detections which may be caused from instances such as from fog, different lighting conditions caused by nature, and other external optical effects.

\section{REFERENCES}

[1] M. Li, W. Xu, K. Xu, J. Fan, and D. Hou, "Review of fire detection technologies based on video image", JATIT, vol. 49, no. 2, pp. 700-707, Mar. 2013.

[2] W. Wenhao, Z. Hong, " Fire detection based on flame color and area” ,2012 IEEE Int. Conf. on Computer Science and Automation Engineering, pp. 222-226, 2012.

[3] C. Juan, H. Yaping, W. Jian, “ Multi-feature fusion based fast video flame detection", Building and Environment, vol. 45, pp. 1113$1122,2010$.

[4] T. Celik, and H. Demirel, “ Fire detection in video sequences using a generic color model", Fire Safety J, 2008, doi:10.1016/j.firesaf.2008.05.005

[5] Z. Junying, and D. Xiaoxiao, " Image recognition technology in fire detection", Fire Science and Technology, vol. 26, no. 4, pp. 417420, 2007.

[6] Y. Y. Yan, S. B. Gao, H. Y. Wang, and Z. B. Guo, “ Contour extraction of flame for fire detection", Advanced Materials Research, Manufacturing Science and Technology, vol, 383-390, pp. 1106-1110, 2012.

[7] J. Rong, D. Zhou, W. Yao, W. Gao, J. Chen, and J. Wang, “ Fire flame detection based on GICA and target tracking", Optics \& Laser Technology, vol. 47, pp. 283-291, 2013.

[8] W. Lei, and J. Liu, " Early fire detection in coalmine based on video processing", Advances in Intelligent Systems and Computing, vol. 181, pp. 239-245, 2013

[9] T. Celik, "Fast and efficient method for fire detection using image processing” , ETRI Journal, vol. 32, no. 6, pp. 811-890, 2010.

[10] Jareerat Seebamrungsat, Suphachai Praising, and Panomkhawn Riyamongkol," Fire Detection in buildings using image processing",2014.

[11] R. C. Gonzalez, and R. E. Wood, "Digital image processing", Prentice Hall, 3rd edition, 2007.

[12] R. C. Gonzalez, R. E. Wood, and S. L. Eddins, “ Digital image processing using Matlab, Gatemark Publishing, 2nd edition, 2007 